\title{
Successful treatment of granulomatosis with polyangiitis with hydropneumothorax using corticosteroids and immunosuppressant
}

\author{
XU-HUA SHI, YONG-FENG ZHANG and YUE-WU LU \\ Department of Rheumatology and Immunology, Beijing Chao-Yang Hospital, \\ Capital Medical University, Beijing 100020, P.R. China \\ Received February 3, 2016; Accepted February 10, 2017
}

DOI: $10.3892 /$ etm.2017.4440

\begin{abstract}
Pneumothorax and pleural effusion is a rare and serious complication of granulomatosis with polyangiitis (GPA). The present study reported a case with a history of sinusitis for 20 years, dry cough for three years and exacerbated purulent nasal discharge and recurrent skin ulcers for two years. The patient experienced sudden difficulty in breathing two months prior to presentation. Lung computed tomography (CT) showed multiple bilateral lung nodules and cavitary nodules as well as right hydropneumothorax. Paranasal sinus CT showed soft tissue infiltration. The cytoplasmic pattern of anti-neutrophil cytoplasmic antibody (c-ANCA) was positive and anti-proteinase 3 (PR3) antibodies, erythrocyte sedimentation rate and $\mathrm{C}$-reactive protein were elevated. After pleural drainage and methylprednisolone pulse treatment, followed by cyclophosphamide and cyclosporine, the patient's symptoms were ameliorated, lungs were re-expanded, and c-ANCA, PR3 and inflammatory markers returned to normal levels.
\end{abstract}

\section{Introduction}

Granulomatosis with polyangiitis (GPA) is a necrotizing granulomatous inflammation with multi-organ involvement, including respiratory system-necrotizing granulomas and glomerulonephritis. Multiple pulmonary nodules, pulmonary infiltrates and pulmonary alveolar hemorrhage are common manifestations in the lung. Although respiratory tract involvement is present in $61-94 \%(1,2)$, pleural involvement occurs in $<10 \%$ of cases (3). Pleurisy, pleural effusion and

Correspondence to: Dr Yue-Wu Lu, Department of Rheumatology and Immunology, Beijing Chao-Yang Hospital, Capital Medical University, 8 Gongren Tiyuchang Nanlu, Chaoyang, Beijing 100020, P.R. China

E-mail: sxhherosci@sina.com

Key words: Wegener's granulomatosis, with polyangiitis, pneumothorax, therapy, corticosteroids, immunosuppressant pleural thickening are common in pleural involvement (4-6). Pneumothorax or hydropneumothorax are rare but serious complications, which often result in secondary infection, respiratory failure and death (7-9). The present study reported on a case of GPA with hydropneumothorax. The patient successfully recovered after corticosteroid and immunosuppressant treatments.

\section{Case report}

A 43-year-old man, who had intermittent purulent nasal discharge for 20 years had been diagnosed with 'rhinitis/sinusitis' at external hospitals. The patient had been having a cough, mainly dry cough, for 3 years and had hemoptysis once 3 years ago. The purulent nasal discharge exacerbated 2 years previously, accompanied with nasal granuloma-like discharge, recurrent skin ulcers and scar formation on face and lower limbs (Fig. 1). This patient had received treatment at several hospitals. His lung computed tomography (CT) scan, performed 2 years prior to hospitalization, showed multiple pulmonary nodules. He had been diagnosed with lung infection, but antibiotics were ineffective. He had also been diagnosed with tuberculosis 18 months ago, but treatment for tuberculosis (rifampicin + isoniazid + ethambutol + pyrazinamide) was ineffective. Position emission tomography/CT, performed $\sim 18$ months ago, showed no indications of malignancies. Lung biopsy showed chronic granulomatous inflammation. Nasal mucosa biopsy showed chronic inflammation. Repeated lung CT 1 year prior to administration to our hospital demonstrated that multiple pulmonary nodules remained (Fig. 2). Two months prior to admission, the patient experienced violent coughs and difficult breathing. He also had fevers, blurred vision, tinnitus, hearing loss and temperature fluctuations of $37.0-38.0^{\circ} \mathrm{C}$. Lung CT showed multiple lung nodules or cavitary nodules. The empty cysts at the right pleura ruptured and formed hydropneumothorax (Fig. 3).

Due to ineffective treatment at external hospitals, the patient was transferred to the Department of Rheumatology and Immunology of Beijing Chao-Yang Hospital (Beijing, China) in August 2015. Physical examination in semi-supine position provided the following results: Blood pressure, 


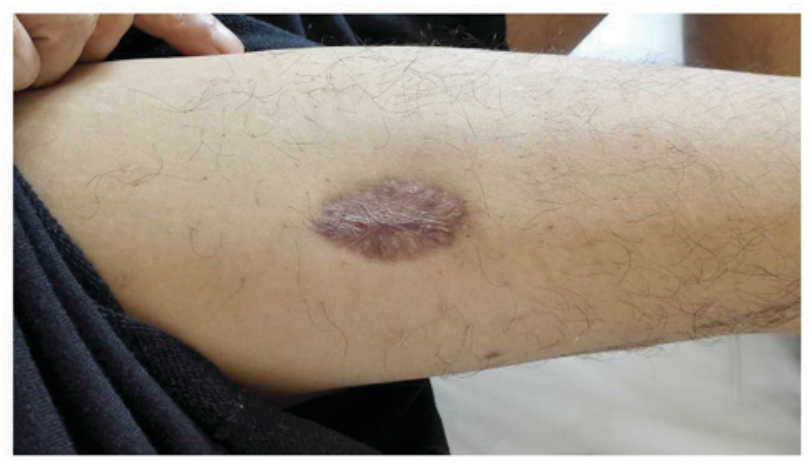

Figure 1. Scar formation after skin ulcer.

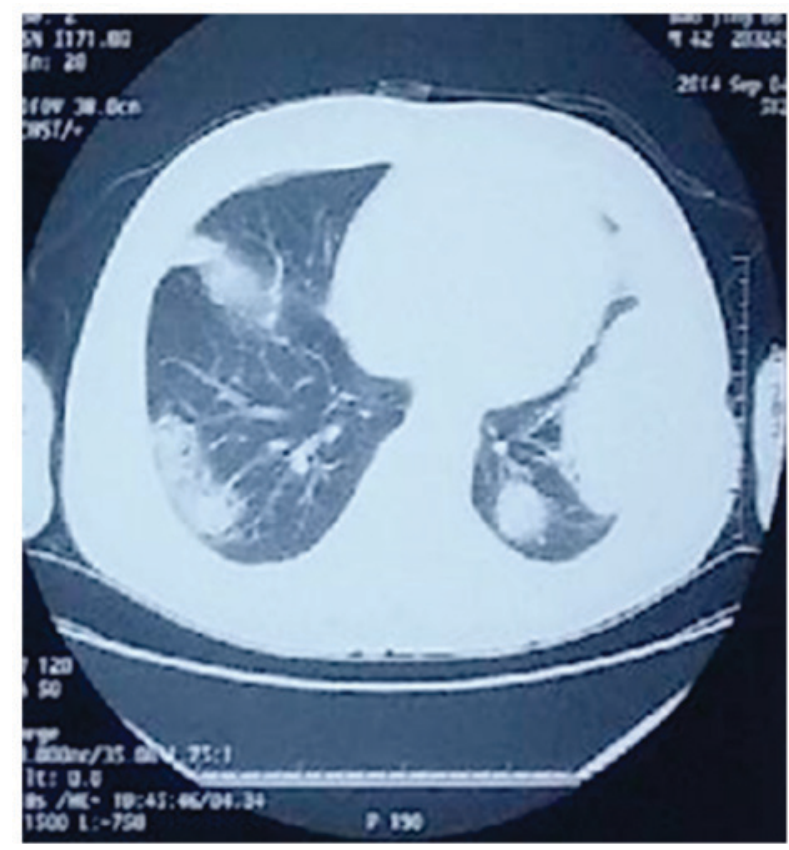

Figure 2. Lung computed tomography scan showing bilateral multiple lung nodules, performed in September 2014.

120/80 mmHg; saddle nose; visible ulceration-induced scars at face and extremities; no breathing sound at the right lung; heart rate, 100 beats/min; regular heartbeat; no abdominal abnormalities. Blood analysis showed increased leukocytes and thrombocytosis. Urine test results were normal. Arterial blood gas results were as follows: Partial pressure of oxygen, $66.3 \mathrm{mmHg}$ (normal range, $83-108 \mathrm{mmHg}$ ); oxygen saturation, 93.3\% (normal range, 95-98\%); erythrocyte sedimentation rate (ESR), $73 \mathrm{~mm} / \mathrm{h}$ (normal range, $0-20 \mathrm{~mm} / \mathrm{h}$ ); C-reactive protein (CRP), $16 \mathrm{mg} / \mathrm{dl}$ (normal range, 0-0.8 mg/dl); cytoplasmic pattern of anti-neutrophil cytoplasmic antibody (c-ANCA) positive; anti-proteinase 3 (PR3) antibodies, $150.50 \mathrm{RU} / \mathrm{ml}$ (normal range, 0-25 RU/ml; Table I). Sinus CT showed visible soft tissue infiltration at the bilateral maxillary, ethmoid and sphenoid sinus; bone destruction and absorption at the inner portion of the left maxillary and ethmoid sinus (Fig. 4). Lung CT showed that the mediastinum had turned to the left side, multiple lung nodules or cavitary nodules, a subpleural nodule ruptured into the right pleural cavity to form a hydropneumothorax and bilateral pleural thickening (Fig. 5A).

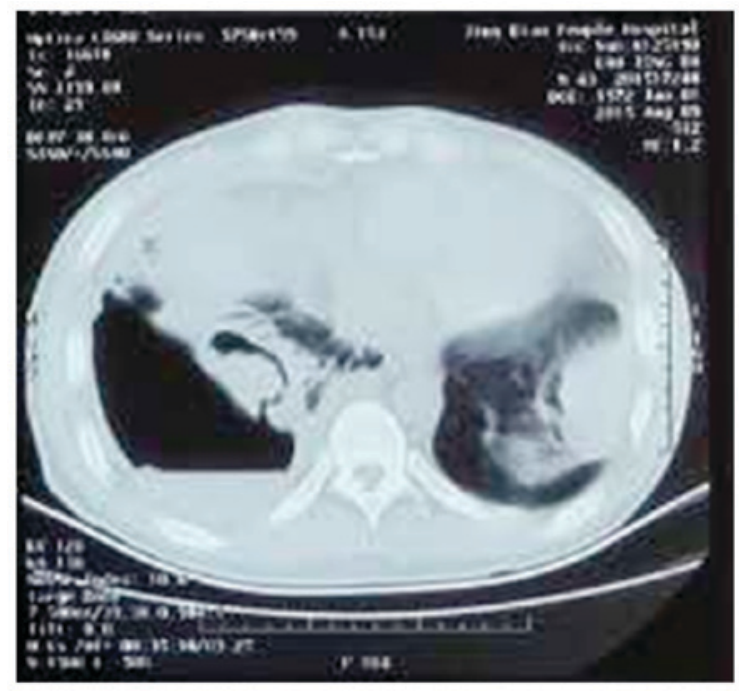

Figure 3. The right pleural nodules ruptured into the pleural cavity, forming hydropneumothorax, as shown in a lung computed tomography scan performed in July 2015.

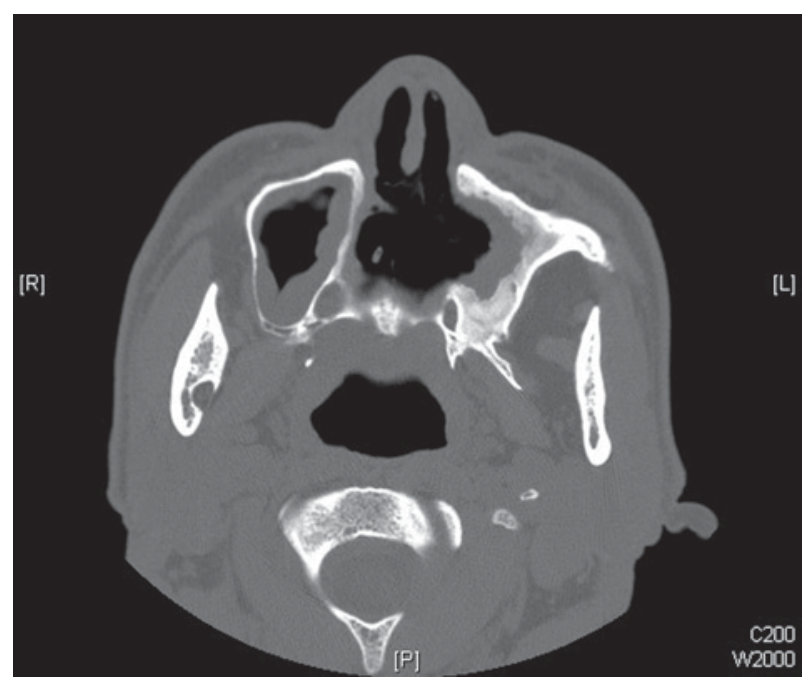

Figure 4. Paranasal sinus computed tomography scan showing sinus soft tissue infiltration, performed in August 2015. Part of the sinus wall was destroyed.

According to the 1990 American College of Rheumatology classification criteria (10), this patient was diagnosed with 'GPA with hydropneumothorax'. The patient was treated with methylprednisolone (48 mg/day), cyclophosphamide (0.4 g/week intravenously) and pleural drainage. Sputum culture and pleural fluid culture indicated that Aspergillus niger was positive. The patient's symptoms ameliorated after corticosteroid and immunosuppressant treatment, c-ANCA, ESR and CRP returned to normal levels and PR3 levels were decreased (Table I). After consultation with the Department of Infectious Diseases of Beijing Chao-Yang Hospital (Beijing, China), the infestation of Aspergillus niger was classified as colonization; therefore, the patient did not receive any antibiotic treatments. After 3 weeks of the treatments, lung CT indicated that pneumothorax was reduced; however, the lung did not fully re-expand and the volume of pulmonary nodules was slightly decreased (Fig. 5B). The 


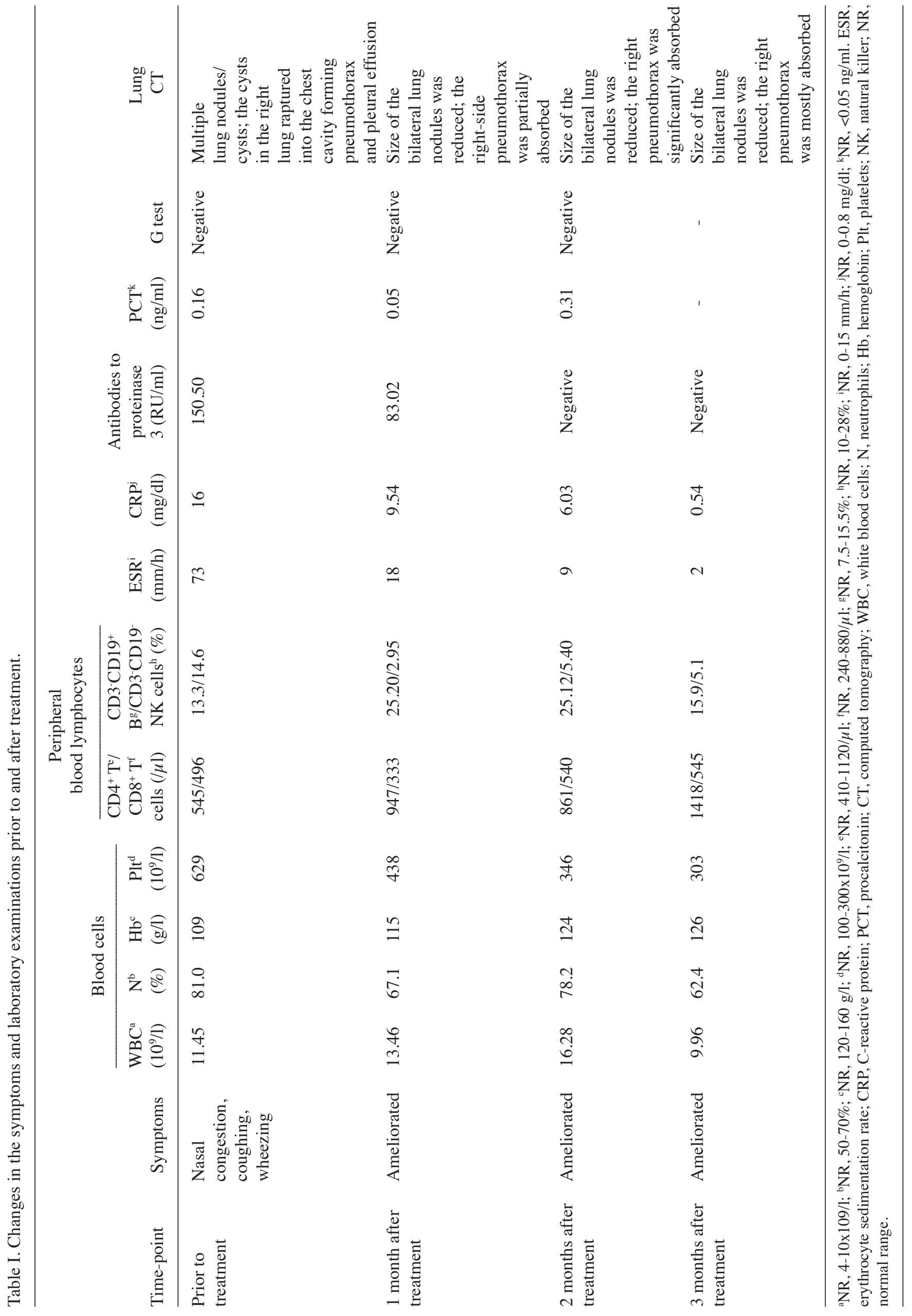



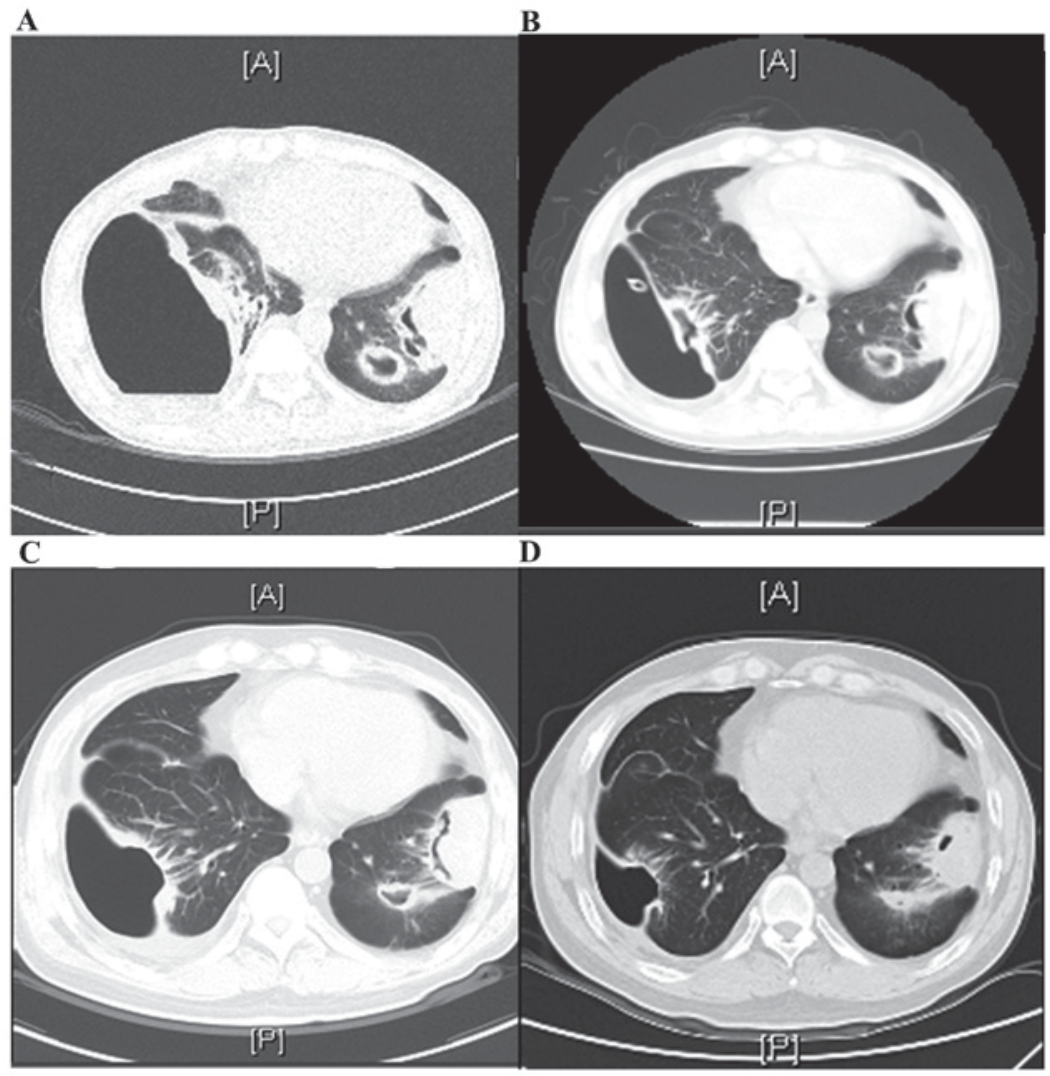

Figure 5. Changes in the lung computed tomography images prior to and after treatment. (A) Prior to treatment (August 2015) and at (B) 1 month (September 2015) (C) 2 months (October 2015) and (D) 3 months (November 2015) after treatment. A, anterior; P, posterior.

patient was treated with a methylprednisolone pulse of $1 \mathrm{~g}$ per day for 3 days, returned to the original dose, followed by gradual dose reduction, combined with cyclosporin A (100 mg). The thoracic drainage tube was removed 4 days after the pulse treatment. At the follow-up visit 2 months after the treatment, the patient did not have any symptoms, ESR and CRP were normal and c-ANCA was negative (Table I). Lung CT showed an improvement of lung lesions (Fig. 5C). At the follow-up visit 3 months after the treatment, methylprednisolone was reduced to $12 \mathrm{mg} / \mathrm{day}$; the patient did not show any symptoms, ESR and CRP were normal, and ANCA was negative (Table I). Lung CT showed further improvement, right pneumothorax was almost absorbed and the subpleural cavity nodules disappeared (Fig. 5D).

\section{Discussion}

Pulmonary involvement is common in granulomatous vasculitis and may be the first manifestation of this disease (1). Single or multiple nodules of varying sizes are common manifestations of lung disease, accompanied with nodule necrosis and cavitation. The necrosis and rupture of the subpleural nodules may cause pneumothorax and pleural effusion. Pneumothorax and pleural effusion are rare and serious complications. Patients often die from respiratory failure or infection due to the increased risk of infection $(2,3,11)$.

The causes of pneumothorax in GPA include cavitied nodule rupture, pleural involvement, infection as well as corticosteroids and immunosuppressive therapy $(4,6,11-18)$.
The patient of the present case study did not receive effective treatment prior to the occurrence of pneumothorax. He had multiple pulmonary nodules and cavitation, serious lesions in sinuses and skin, c-ANCA was positive and ANCA, ESR and CRP levels were elevated, indicating the disease was in the active phase. At the side of the pneumothorax, the cavitied nodules were located near the pleura, accompanied with pleural thickening. Aspergillus niger was positive in the sputum and pleural drainage culture. Growth of microorganisms in cavitied nodules may cause tissue fragility. Likely due to this risk factor, a subpleural nodule ruptured into the right pleural cavity, which resulted in pneumothorax.

The patient's sputum culture and chest drainage culture were positive for Aspergillus niger but negative for bacteria, tuberculosis and pneumocystis carinii. The patient's symptoms were quickly relieved after corticosteroid and immunosuppressive therapy. As after consultation with the Department of Infectious Diseases of Beijing Chao-Yang Hospital (Beijing, China), the presence of Aspergillus niger was characterized as a colonization, the patient was not treated with antibiotics. The patient's symptoms quickly disappeared after corticosteroid and immunosuppressive therapy, and the ANCA, ESR and CRP returned to normal levels. The patient's condition was stable in the subsequent follow-up visits at two and three months. Studies have shown that infection is a risk factor of pneumothorax in patients with granulomatous vasculitis and is a common complication and cause of death in patients receiving immunosuppressive therapy $(11,14,15)$. Although Aspergillus niger was detected in the patient, subsequent 
observation of the response to the drug for GPA indicated that the presence of Aspergillus niger was characterized as colonization, rather than current infection. This judgement was further confirmed during long-term follow-up. Microbial colonization may occur in patients with GPA, therefore treatment decisions should be based on comprehensive assessment of the patient's actual condition rather than laboratory results alone.

Pleural drainage helps drain air, blood or fluid from the pleural space, but its application in the pneumothorax/hydropneumothorax caused by cavity nodule rupture in GPA requires careful evaluation $(3,13,17)$. In the case of the present study, the pneumothorax was absorbed after pleural drainage but the lung did not fully re-expand. The patient's primary disease was not completely controlled; hence, there was no repair of the impaired pleura. Considering the higher risk of secondary infection, the pleural drainage tube was removed after enhanced treatment of the primary disease. At subsequent follow-up visits, it was identified that the remaining pneumothorax was gradually absorbed. It has been shown that pleural drainage is not necessary in patients in a good general physical condition (8). Based on our clinical experience, the decision to perform pleural drainage should be based on the patient's condition. Under non-emergency circumstances, if pleural drainage cannot improve lung re-expansion, the drainage tube should be removed timely to reduce the risk of infection.

Certain studies have shown that corticosteroids and immunosuppressive agents may cause delayed wound healing, fibrosis and increased tissue fragility, which are risk factors of pneumothorax $(17,19)$. In the patient of the present study, the pneumothorax occurred prior to corticosteroid and immunosuppressive treatments. When the pleural drainage did not lead to re-expansion of the lung, immunosuppressive therapy improved the patient's symptoms. As pneumothorax/hydropneumothorax occurs in patients with active disease, early treatment of the primary disease with potent immunosuppressive agents is required to promote lesion healing. Due to the increased risk of infection after application of corticosteroids and immunosuppressants (2), the patient's symptoms, procalcitonin levels and lymphocyte function were closely monitored. As the patient did not have any infection, the disease was controlled and its status sustained by continued immunosuppressive therapy. It is suggested that if an infection occurs, suitable antibiotics should be added to the continued immunosuppressive therapy instead of the termination of the therapy for GPA (11). Interruption of the immunosuppressive treatment may cause a relapse of GPA; therefore, corticosteroids and immunosuppressants require adjusting according to a patient's condition as well as infection and immune status (18).

Compared with previously reported cases, the present case is different regarding the following points: i) Although sputum and pleural drainage cultures were positive for Aspergillus niger, its presence was characterized as colonization, not infection; therefore, the patient was not treated with antibiotics; ii) when the lungs did not fully re-expand after pleural drainage, methylprednisolone pulse therapy was applied and immunosuppressive treatment was enhanced, following successful removal of the pleural drainage tube; iii) the patient's symptoms and immune status were closely monitored and infection was effectively prevented. According to our clinical experience, pleural drainage is a supportive means in the treatment of GPA complicated with pneumothorax and should be given or ceased based on the patient's condition. It is most important to completely control the primary disease by potent immunosuppressive agents and closely monitor infections due to increased risk of their occurrence.

\section{References}

1. Abdou NI, Kullman GJ, Hoffman GS, Sharp GC, Specks U, McDonald T, Garrity J, Goeken JA and Allen NB: Wegener's granulomatosis: Survey of 701 patients in North America. Changes in outcome in the 1990s. J Rheumatol 29: 309-316, 2002.

2. Sezer I, Kocabas H, Melikoglu MA, Budak BS, Ozbudak IH and Butun B: Spontaneous pneumothorax in Wegener's granulomatosis: A case report. Mod Rheumatol 18: 76-80, 2008.

3. Delèvaux I, Khellaf M, André M, Michel JL, Piette JC and Aumaître O: Spontaneous pneumothorax in Wegener granulomatosis. Chest 128: 3074-3075, 2005.

4. Maguire R, Fauci AS, Doppman JL and Wolff SM: Unusual radiographic features of Wegener's granulomatosis. AJR Am J Roentgenol 130: 233-238, 1978.

5. Cordier JF, Valeyre D, Guillevin L, Loire R and Brechot JM: Pulmonary Wegener's granulomatosis. A clinical and imaging study of 77 cases. Chest 97: 906-912, 1990.

6. Belhassen-Garcia M, Velasco-Tirado V, Alvela-Suaréz L, Carpio-Peréz A, Lledías JP, Novoa N, Iglesias-Gómez A and Cordero-Sánchez M: Spontaneous pneumothorax in Wegener's granulomatosis: Case report and literature review. Semin Arthritis Rheum 41: 455-460, 2011.

7. Farrelly CA: Wegener's granulomatosis: A radiological review of the pulmonary manifestations at initial presentation and during relapse. Clin Radiol 33: 545-551, 1982.

8. Shimizu T, Ohara T, Ito S, Nakano M, Tsutsui N, Sato T, Suzuki E and Gejyo F: A case of Wegener's granulomatosis complicated with seropneumothorax. Mod Rheumatol 13: 181-184, 2003.

9. Ates A, Karaaslan Y, Yildiz G, Günesen O and Han S: A case of Wegener's granulomatosis complicated with hydropneumothorax. J Clin Rheumatol 12: 264-265, 2006.

10. Leavitt RY, Fauci AS, Bloch DA, Michel BA, Hunder GG, Arend WP, Calabrese LH, Fries JF, Lie JT, Lightfoot RW Jr, et al: The American College of Rheumatology 1990 criteria for the classification of Wegener's granulomatosis. Arthritis Rheum 33: 1101-1107, 1990.

11. Bülbül Y, Ozlü T and Oztuna F: Wegener's granulomatosis with parotid gland involvement and pneumothorax. Med Princ Pract 12: 133-137, 2003.

12. Gohel VK, Dalinka MK, Israel HL and Libshitz HI: The radiological manifestations of Wegener's granulomatosis. Br J Radiol 46: 427-432, 1973.

13. Epstein DM, Gefter WB, Miller WT, Gohel V and Bonavita JA: Spontaneous pneumothorax: An uncommon manifestation of Wegener granulomatosis. Radiology 135: 327-328, 1980

14. Jaspan T, Davison AM and Walker WC: Spontaneous pneumothorax in Wegener's granulomatosis. Thorax 37: 774-775, 1982.

15. Wolffenbuttel BH, Weber RF and Kho GS: Pyopneumothorax: A rare complication of Wegener's granulomatosis. Eur J Respir Dis 67: 223-227, 1985

16. Ogawa M, Azemoto R, Makino Y, Mori Y, Ueda S, Wakashin M and Ohto M: Pneumothorax in a patient with Wegener's granulomatosis during treatment with immunosuppressive agents. J Intern Med 229: 189-192, 1991.

17. Storelli E, Casali C, Natali P, Rossi G and Morandi U: Unusual pathogenesis of spontaneous pneumothorax secondary to Wegener's granulomatosis. Ann Thorac Surg 84: 288-290, 2007.

18. Kahraman H, Inci MF, Tokur M and Cetin GY: Spontaneous pneumothorax in a patient with granulomatosis with polyangiitis. BMJ Case Rep 2012: pii: bcr2012007765, 2012.

19. Travis WD, Hoffman GS, Leavitt RY, Pass HI and Fauci AS: Surgical pathology of the lung in Wegener's granulomatosis. Review of 87 open lung biopsies from 67 patients. Am J Surg Pathol 15: 315-333, 1991. 